PROCEEDINGS OF THE

AMERICAN MATHEMATICAL SOCIETY

Volume 127, Number 11, Pages 3329-3338

S 0002-9939(99)05105-9

Article electronically published on May 3, 1999

\title{
ASYMPTOTIC EULERIAN EXPANSIONS FOR BINOMIAL AND NEGATIVE BINOMIAL RECIPROCALS
}

\author{
EWA MARCINIAK AND JACEK WESOŁOWSKI
}

(Communicated by Wei-Yin Loh)

\begin{abstract}
Asymptotic expansions of any order for expectations of inverses of random variables with positive binomial and negative binomial distributions are obtained in terms of the Eulerian polynomials. The paper extends and improves upon an expansion due to David and Johnson (1956-7).
\end{abstract}

\section{INTRODUCTION}

If one wants to estimate a mean of a population $Z$ on the basis of a sample $Z_{1}, \ldots, Z_{M}$ of a random length $M$, which is a natural-valued non-zero random variable independent of the sequence $\left(Z_{1}, Z_{2}, \ldots\right)$ of independent random variables with a common distribution equal to that of the random variable $Z$, then the unbiased estimate

$$
T=\frac{1}{M} \sum_{i=1}^{M} Z_{i}
$$

is usually exploited. Its efficiency is measured by the variance (if only $Z$ is square integrable). A straightforward argument based on conditioning leads to the formula

$$
\operatorname{Var}(T)=\sigma^{2} E(1 / M)
$$

where $\sigma^{2}$ is the variance of $Z$. For details and a more thorough study of $T$ and another related estimate one can consult Szabłowski, Wesołowski and Wieczorkowski (SWW) (1996) (or SWW (1997) for a shortened English version).

Then a question of computing the first inverse moment for positive discrete distributions arises. Different general approaches to that problem, including also a related one of inverse factorial moments, can be found in the literature for instance in Chao and Strawderman (1972), Kabe (1976), Cressie, Davis, Folks and Policello (1981), Jones (1987), or Rockower (1988).

In this paper we treat only two special distributions for $M$ : binomial $b(n, p)$ and negative binomial $n b(n, p)$. They appear in such a context in a natural way if one takes a sample with replacement from a given finite population of size $N$ and is interested in estimation of some parameters in a subpopulation $A$ of size $N_{A}$. Then two cases are of potential interest: (i) the size of the sample is $n$ and then the size $n_{A}$ of the subsample falling in $A$ is a random variable with the binomial $b\left(n, N_{A} / N\right)$

Received by the editors January 14, 1998.

1991 Mathematics Subject Classification. Primary 60E05, 62E20; Secondary 11B68, 05 A16.

Key words and phrases. Eulerian numbers, Eulerian polynomials, asymptotic series expansions, inverse moments, positive binomial distribution, positive negative binomial distribution. 
distribution; (ii) elements are drawn up to $n$-th draw from outside of $A$ and then $n_{A}$ has the $n b\left(n, N_{A} / N\right)$ distribution (this is closely related to the inverse sampling technique used in survey sampling methodology (see for instance Cochran (1977), Ch. 4.5)). If in the both situations only samples with non-zero $n_{A}$ 's are considered, then the distributions are truncated at zero.

Let us recall the basic formulas for the two cases:

(i) the binomial $b(n, p)$ distribution truncated at zero, i.e.

$$
P(M=i)=\frac{1}{1-q^{n}}\left(\begin{array}{c}
n \\
i
\end{array}\right) p^{i} q^{n-i}, \quad i=1,2, \ldots, n,
$$

where $n>0$ is a natural number, $p \in(0,1)$ and $q=1-p$; then

$$
E\left(M^{-1}\right)=\frac{1}{1-q^{n}} h_{n}(q)
$$

where

$$
h_{n}(q)=\sum_{i=1}^{n} \frac{1}{i}\left(\begin{array}{c}
n \\
i
\end{array}\right) p^{i} q^{n-i}
$$

(ii) the negative binomial $n b(n, p)$ distribution truncated at zero, i.e.

$$
P(M=i)=\frac{1}{1-q^{n}}\left(\begin{array}{c}
n+i-1 \\
i
\end{array}\right) p^{i} q^{n}, \quad i=1,2, \ldots,
$$

where $n>0$ is again a natural number, $p \in(0,1)$ and $q=1-p$; then

$$
E\left(M^{-1}\right)=\frac{1}{1-q^{n}} g_{n}(q)
$$

where

$$
g_{n}(q)=\sum_{i=1}^{\infty} \frac{1}{i}\left(\begin{array}{c}
n+i-1 \\
i
\end{array}\right) p^{i} q^{n} .
$$

One of the basic questions in estimation is investigation of relations between sample size and efficiency of adopted estimates. In our setting this can be reduced to analyzing the behaviour of $h_{n}$ and $g_{n}$, which are quite complicated functions of $n$. Then instead of looking at exact formulas it could be very useful to find asymptotic approximations of $h_{n}$ and $g_{n}$ for large values of $n$.

This was our way to come to the asymptotic formulas, which are the main results of the paper, but we believe that the expansions we provide could be of some interest to people from other areas of mathematics, and, first of all, to specialists in discrete methods. The main results are given in Section 2, while the proofs are put off to Section 3 .

In both cases the expansions are given in terms of the Eulerian polynomials $A_{n}$ (see Foata and Schützenberger (1970) for a thorough study of this subject) defined by the generating function

$$
\frac{1-t}{1-t \exp [(1-t) u]}=\sum_{n=0}^{\infty} A_{n}(t) \frac{u^{n}}{n !} .
$$

The $n$-th polynomial has the form $A_{n}(t)=\sum_{k=1}^{n} A_{n, k} t^{k}$, where $A_{n, k}$ denote the classical Eulerian numbers - number of permutations of the set $\{1, \ldots, n\}$ with $k$ descents. They are defined by the recurrence formula:

$$
A_{n, k}=k A_{n-1, k}+(n-k+1) A_{n-1, k-1}, \quad n=2,3, \ldots, k=2 \ldots, n,
$$


where the recurrence starts with $A_{1, k}=0, k=2, \ldots$, and $A_{n, 1}=1, n=1,2, \ldots$ (see for instance Graham, Knuth, Patashnik (1994) (Ch. 6.2)). The Eulerian triangle has the form

$\begin{array}{ccccccll}: & 1 & 2 & 3 & 4 & 5 & 6 & k \\ 1 & 1 & : & : & : & : & : & : \\ 2 & 1 & 1 & : & : & : & : & : \\ 3 & 1 & 4 & 1 & : & : & : & : \\ 4 & 1 & 11 & 11 & 1 & : & : & : \\ 5 & 1 & 26 & 66 & 26 & 1 & : & : \\ 6 & 1 & 57 & 302 & 302 & 57 & 1 & : \\ n & : & : & : & : & : & : & :\end{array}$

The basic property of the Eulerian polynomials we will exploit is the recurrence formula

$$
A_{n}(t)=\sum_{i=0}^{n-1}\left(\begin{array}{c}
n \\
i
\end{array}\right) A_{i}(t) t(1-t)^{n-i-1}
$$

where $A_{0}(t)=1$ - see, for instance, (2.6) in a recent contribution by Désarménien and Foata (1995).

Let us point out that the inverse moments of the positive binomial distributions were quite intensively studied in the literature up to the seventies. First approximate computational formulas go back to Stephan (1946). Tiago de Oliveira (1952) derived the formula we give in Lemma 2 below via some differential equation for $E\left(M^{-1}\right)$ as a function of $q$. Then some exact tables were constructed by Grab and Savage (1954), together with pointing to a possible approximation of $E\left(M^{-1}\right)$ by $1 /(n p-q)$.

In David and Johnson (1956-7) a new approximating formula was given:

$$
E\left(M^{-1}\right) \approx \frac{1}{n p}\left(1+\frac{q}{n p}+\frac{q(q+1)}{(n p)^{2}}+\frac{q\left(q^{2}+4 q+1\right)}{(n p)^{3}}+\frac{q\left(q^{3}+11 q^{2}+11 q+1\right)}{(n p)^{4}}\right),
$$

which looks like ours for $k=5$ (see Theorem 1 below): observe that looking at the Eulerian triangle we can easily rewrite the above formula as

$$
E\left(M^{-1}\right) \approx \sum_{j=0}^{4} \frac{A_{j}(q)}{(n p)^{j+1}} .
$$

Unfortunately the derivation given in David and Johnson (1956-7) is rather informal; as the authors write it is "not to be regarded as initial terms in expansions for the reciprocal mean (variance), but as approximation formulae", and the quality of approximation is checked only computationally. One of the consequences of the present paper is that the theoretical background for this formula is provided.

Another approximation, using the beta function approach, by

$$
(n-2) /\{n[(n-1) p-1]\}
$$

was suggested in Mendenhall and Lehman, Jr. (1960), together with a possible application in life testing (see also Patel and Gajjar (1995) for a recent contribution). Then some recurrence relations for $E\left(M^{-1}\right)$ were studied in Govindarajulu (1963) and an approximation by $1 /(n p)$ was more thoroughly studied in Thionet (1963) (here the mathematical rigourousness was preserved); see also Rempala and 
Székely (1998) for a very recent contribution containing a new approximation of order $O\left(n^{-3}\right)$. In Stancu (1968) an exact formula for $E\left(M^{-1}\right)$ in terms of an infinite sum of some difference operators was given. This direction was further developed in Kabe (1976). The special case of our Theorem 1 for $k=2$ has been recently derived in SWW (1996).

\section{EXPANSIONS}

Begin with analyzing the asymptotic properties of $h_{n}(q)$ defined in (1) as $n \rightarrow \infty$. The main result brings approximations of $h_{n}(q)$ by finite linear combinations of powers of $1 / n$ :

Theorem 1. For any $k=1,2, \ldots$

$$
\sum_{i=1}^{n} \frac{1}{i}\left(\begin{array}{c}
n \\
i
\end{array}\right) p^{i} q^{n-i}=\sum_{j=0}^{k-1} \frac{A_{j}(q)}{p^{j+1}} \frac{1}{n^{j+1}}+o\left((1 / n)^{k}\right) .
$$

Inserting, for instance, $p=q=1 / 2$ in the formula (5) and computing respective coefficients we immediately get the following simple expansions:

$$
\begin{gathered}
\sum_{i=1}^{n} \frac{1}{i}\left(\begin{array}{c}
n \\
i
\end{array}\right)=2^{n}\left(\frac{2}{n}+o(1 / n)\right) \\
=2^{n}\left(\frac{2}{n}+\frac{2}{n^{2}}+o\left(1 / n^{2}\right)\right) \\
=2^{n}\left(\frac{2}{n}+\frac{2}{n^{2}}+\frac{6}{n^{3}}+o\left(1 / n^{3}\right)\right) \\
=2^{n}\left(\frac{2}{n}+\frac{2}{n^{2}}+\frac{6}{n^{3}}+\frac{26}{n^{4}}+o\left(1 / n^{4}\right)\right),
\end{gathered}
$$

which, we believe, could be valuable companions of classical asymptotic approximations, reviewed, for instance, in Ch. 9.3 of Graham, Knuth Patashnik (1994).

Observe that since the left-hand side of (3) for $t=q \in(0,1)$ is the moment generating function of the random variable $p Y$, where $p=1-q$ and $Y$ is a random variable with the geometric $n b(1, p)$ distribution, then alternatively the expansion can be rewritten as

$$
\sum_{i=1}^{n} \frac{1}{i}\left(\begin{array}{c}
n \\
i
\end{array}\right) p^{i} q^{n-i}=\frac{1}{p} \sum_{j=0}^{k-1} \frac{E\left(Y^{j}\right)}{n^{j+1}}+o\left((1 / n)^{k}\right) .
$$

The proof of Theorem 1, based on the Stolz theorem and the recurrence (4) for the Eulerian polynomials, is given in Section 3.

To pass from the result for the binomial case to the related one for the negative binomial case (2) it is convenient to note an identity involving both $h_{n}$ and $g_{n}$, which seems to be of some interest, also in view of the asymptotic behaviour of the difference $h_{n}-g_{n}$.

Lemma 1. For any $n=1,2, \ldots$ and any $q \in(0,1)$

$$
g_{n}(q)=h_{n}(q)-1 / n-q^{n}(\log (q)-1 / n),
$$

where $h_{n}$ and $g_{n}$ are defined in (1) and (2), respectively. 
Proof. We use the induction with respect to $n$. For $n=1$ (6) follows directly from the Maclaurin expansion of the log fuction. Now assume that (6) holds for some $n=m-1 \geq 1$ and consider $n=m$. Then applying the reccurence formula for the binomial coefficients

$$
\left(\begin{array}{c}
m \\
i
\end{array}\right)=\left(\begin{array}{c}
m-1 \\
i
\end{array}\right)+\left(\begin{array}{c}
m-1 \\
i-1
\end{array}\right)
$$

the identity

$$
\frac{1}{i}\left(\begin{array}{c}
m+i-2 \\
i-1
\end{array}\right)=\frac{1}{m-1}\left(\begin{array}{c}
m+i-2 \\
i
\end{array}\right),
$$

and the induction assumption, we get

$$
g_{m}(q)=q\left(h_{m-1}(q)-q^{m-1} \log (q)\right) .
$$

Now (7) and the identity

$$
\frac{1}{i}\left(\begin{array}{c}
m-1 \\
i-1
\end{array}\right)=\frac{1}{m}\left(\begin{array}{c}
m \\
i
\end{array}\right)
$$

imply

$$
q h_{m-1}(q)=h_{m}(q)-\left(1-q^{m}\right) / m .
$$

The above identity (6) allows us to conclude the approximation in the negative binomial case (2) directly from Theorem 1 . The expansion needs only a slight modification of one of the coefficients from (5).

Theorem 2. For any $k=1,2, \ldots$

$$
\sum_{i=1}^{\infty} \frac{1}{i}\left(\begin{array}{c}
n+i-1 \\
i
\end{array}\right) p^{i} q^{n}=\sum_{j=0}^{k-1} \frac{A_{j}(q)}{p^{j+1}} \frac{1}{n^{j+1}}-\frac{1}{n}+o\left(1 / n^{k}\right) .
$$

Proof. It follows immediately from Lemma 1 and Theorem 1.

Now we can give as a simple conclusion some explicit expansions, taking for instance $p=q=1 / 2$ as in the binomial case. Then directly from Theorem 2 one has:

$$
\begin{gathered}
\sum_{i=1}^{\infty} \frac{1}{i: 2^{i}}\left(\begin{array}{c}
n+i-1 \\
i
\end{array}\right)=2^{n}\left(\frac{1}{n}+o(1 / n)\right) \\
=2^{n}\left(\frac{1}{n}+\frac{2}{n^{2}}+o\left(1 / n^{2}\right)\right) \\
=2^{n}\left(\frac{1}{n}+\frac{2}{n^{2}}+\frac{6}{n^{3}}+o\left(1 / n^{3}\right)\right) \\
=2^{n}\left(\frac{1}{n}+\frac{2}{n^{2}}+\frac{6}{n^{3}}+\frac{26}{n^{4}}+o\left(1 / n^{4}\right)\right)
\end{gathered}
$$

Again, similarly as in the binomial case we can insert $A_{j}(q)=p^{j} E\left(Y^{j}\right), j=$ $0,1, \ldots$, where $Y$ is a geometric $n b(1, p)$ random variable, in (8) obtaining the following form of the expansion:

$$
\sum_{i=1}^{\infty} \frac{1}{i}\left(\begin{array}{c}
n+i-1 \\
i
\end{array}\right) p^{i} q^{n}=\frac{1}{p} \sum_{j=0}^{k-1} \frac{E\left(Y^{j}\right)}{n^{j+1}}-\frac{1}{n}+o\left(1 / n^{k}\right) .
$$




\section{Auxiliary Results AND PRoofs}

Let us begin with rewriting the formula for $h_{n}$.

Lemma 2. For any $n=1,2, \ldots$ and any $q \in(0,1)$

$$
h_{n}(q)=\sum_{i=1}^{n} \frac{q^{n-i}}{i}-q^{n} \sum_{i=1}^{n} \frac{1}{i} .
$$

The proof of Lemma 2 uses a straightforward induction argument based on the recurrence formula for the binomial coefficients (7) and is skipped. Another neat derivation can be found in Tiago de Oliveira (1952), as has been already mentioned in Section 1.

Observe that the second part of the formula (9), i.e. the expression

$$
q^{n} \sum_{i=1}^{n} \frac{1}{i}=o\left(1 / n^{k}\right)
$$

for any $k=1,2, \ldots$. Consequently in the sequel we consider only the first part of (9).

Lemma 3. Define for any $n=1,2, \ldots$

$$
a_{n}^{(0)}=n \sum_{i=1}^{n} \frac{q^{n-i}}{i}, \quad a_{n}^{(k)}=n\left(a_{n}^{(k-1)}-\gamma_{k-1}\right), \quad k=1,2, \ldots,
$$

where the sequence $\left(\gamma_{k}\right)_{k=0,1, \ldots}$ is defined by

$$
\gamma_{0}=\frac{1}{p}, \quad \gamma_{k}=\frac{q}{p} \sum_{i=0}^{k-1}\left(\begin{array}{c}
k \\
i
\end{array}\right) \gamma_{i}, \quad k=1,2, \ldots
$$

Then

$$
\lim _{n \rightarrow \infty} a_{n}^{(k)}=\gamma_{k}, \quad k=0,1, \ldots
$$

Proof. In the first step of the proof we show that the following relations hold:

$$
a_{n}^{(k)}=q \sum_{i=0}^{k}\left(\begin{array}{c}
k \\
i
\end{array}\right) a_{n-1}^{(i)}+a_{n}^{(k-1)}-\gamma_{k-1}, \quad k=1,2, \ldots,
$$

for any $n=2,3, \ldots$.

To prove (11) we use an induction argument with respect to $k$. First let us take $k=1$. Then from the definition of $a_{n}^{(0)}$ and the recurrence relation one obtains

$$
\begin{aligned}
a_{n}^{(1)}= & n\left(a_{n}^{(0)}-\gamma_{0}\right)=(n-1)\left(n \sum_{i=1}^{n} \frac{q^{n-i}}{i}-\gamma_{0}\right)+a_{n}^{(0)}-\gamma_{0} \\
= & (n-1)\left(n \sum_{i=1}^{n-1} \frac{q^{n-i}}{i}+1-\gamma_{0}\right)+a_{n}^{(0)}-\gamma_{0} .
\end{aligned}
$$

Obviously

$$
\gamma_{0}=q \gamma_{0}+1
$$


Hence, continuing the above sequence of identities, we get

$$
\begin{aligned}
a_{n}^{(1)} & =(n-1)\left((n-1) \sum_{i=1}^{n-1} \frac{q^{n-i}}{i}+\sum_{i=1}^{n-1} \frac{q^{n-i}}{i}+1-q \gamma_{0}-1\right)+a_{n}^{(0)}-\gamma_{0} \\
& =(n-1)\left(q a_{n-1}^{(0)}+\sum_{i=1}^{n-1} \frac{q^{n-i}}{i}-q \gamma_{0}\right)+a_{n}^{(0)}-\gamma_{0}=q a_{n-1}^{(1)}+q a_{n-1}^{(0)}+a_{n}^{(0)}-\gamma_{0}
\end{aligned}
$$

which is exactly (11) for $k=1$.

Now assume that (11) holds for some $k=m-1 \geq 1$. We will prove that then it holds also for $k=m$. Observe that the definition of $a_{n}^{(m)}$ and the induction assumption imply

$a_{n}^{(m)}=(n-1)\left(q \sum_{i=0}^{m-1}\left(\begin{array}{c}m-1 \\ i\end{array}\right) a_{n-1}^{(i)}+a_{n}^{(m-2)}-\gamma_{m-2}-\gamma_{m-1}\right)+a_{n}^{(m-1)}-\gamma_{m-1}$.

Since the definition of $\gamma_{m-1}$ implies that

$$
\gamma_{m-1}=q \sum_{i=0}^{m-1}\left(\begin{array}{c}
m-1 \\
i
\end{array}\right) \gamma_{i}
$$

then

$$
\begin{aligned}
a_{n}^{(m)}= & (n-1)\left(q \sum_{i=0}^{m-1}\left(\begin{array}{c}
m-1 \\
i
\end{array}\right) a_{n-1}^{(i)}+a_{n}^{(m-2)}\right. \\
& \left.\quad-\gamma_{m-2}-q \sum_{i=0}^{m-1}\left(\begin{array}{c}
m-1 \\
i
\end{array}\right) \gamma_{i}\right)+a_{n}^{(m-1)}-\gamma_{m-1} \\
= & q \sum_{i=0}^{m-1}\left(\begin{array}{c}
m-1 \\
i
\end{array}\right)(n-1)\left(a_{n-1}^{(i)}-\gamma_{i}\right) \\
& +a_{n}^{(m-1)}+(n-1)\left(a_{n}^{(m-2)}-\gamma_{m-2}\right)-\gamma_{m-1} .
\end{aligned}
$$

Again using the induction assumption for $a_{n}^{(m-1)}$ and the definition of $a_{n-1}^{(i)}$ we get

$$
\begin{aligned}
a_{n}^{(m)}= & q \sum_{i=0}^{m-1}\left(\begin{array}{c}
m-1 \\
i
\end{array}\right) a_{n-1}^{(i+1)}+q \sum_{i=0}^{m-1}\left(\begin{array}{c}
m-1 \\
i
\end{array}\right) a_{n-1}^{(i)} \\
& +a_{n}^{(m-2)}-\gamma_{m-2}+(n-1)\left(a_{n}^{(m-2)}-\gamma_{m-2}\right)-\gamma_{m-1} .
\end{aligned}
$$

Now we renumerate the first sum and then use (7) to get the final result (11):

$$
\begin{gathered}
a_{n}^{(m)}=q \sum_{i=1}^{m}\left(\begin{array}{c}
m-1 \\
i-1
\end{array}\right) a_{n-1}^{(i)}+q \sum_{i=0}^{m-1}\left(\begin{array}{c}
m-1 \\
i
\end{array}\right) a_{n-1}^{(i)}+n\left(a_{n}^{(m-2)}-\gamma_{m-2}\right)-\gamma_{m-1} \\
=q \sum_{i=0}^{m}\left(\begin{array}{c}
m \\
i
\end{array}\right) a_{n-1}^{(i)}+a_{n}^{(m-1)}-\gamma_{m-1},
\end{gathered}
$$

which ends the first step of the proof. 
The second part of the proof makes use of the celebrated

Stolz Theorem. Let $\left(c_{n}\right)_{n \geq 1}$ and $\left(d_{n}\right)_{n \geq 1}$ be two sequences of real numbers. If $d_{n} \rightarrow \infty$ as $n \rightarrow \infty$ and $\exists n_{0}$ such that $\forall n>n_{0}, d_{n+1}>d_{n}$, then

$$
\lim _{n \rightarrow \infty} \frac{c_{n}}{d_{n}}=\lim _{n \rightarrow \infty} \frac{c_{n}-c_{n-1}}{d_{n}-d_{n-1}}
$$

if any of the limits exists.

The induction argument with respect to $k$ is used to prove (10). To use the Stolz theorem for $k=0$ observe that

$$
a_{n}^{(0)}=\frac{\sum_{i=1}^{n} \frac{1}{i q^{i}}}{\frac{1}{n q^{n}}}=\frac{c_{n}}{d_{n}} .
$$

Then

$$
\frac{c_{n}-c_{n-1}}{d_{n}-d_{n-1}}=\frac{\frac{1}{n q^{n}}}{\frac{1}{n q^{n}}-\frac{1}{(n-1) q^{n-1}}}=\frac{1}{1-\frac{n q}{n-1}} \stackrel{n \rightarrow \infty}{\longrightarrow} \frac{1}{p}
$$

Consequently (10) for $k=0$ follows from the Stolz theorem.

Now assume that (10) holds for $k=m-1$. Then, following the definition, rewrite $a_{n}^{(m)}$ as

$$
a_{n}^{(m)}=\frac{q^{-n}\left(a_{n}^{(m-1)}-\gamma_{m-1}\right)}{\frac{1}{n q^{n}}}=\frac{c_{n}}{d_{n}}
$$

Consequently

$$
\frac{c_{n}-c_{n-1}}{d_{n}-d_{n-1}}=\frac{n\left(a_{n}^{(m-1)}-\gamma_{m-1}-q a_{n-1}^{(m-1)}+q \gamma_{m-1}\right)}{1-\frac{n q}{n-1}}
$$

For the numerator we have

$c_{n}-c_{n-1}=n\left(a_{n}^{(m-1)}-\gamma_{m-1}\right)-q n\left(a_{n-1}^{(m-1)}-\gamma_{m-1}\right)=a_{n}^{(m)}-q a_{n-1}^{(m)}-q\left(a_{n-1}^{(m-1)}-\gamma_{m-1}\right)$.

Now applying (11) we get

$$
\frac{c_{n}-c_{n-1}}{d_{n}-d_{n-1}}=\frac{q \sum_{i=0}^{m-1}\left(\begin{array}{c}
m \\
i
\end{array}\right) a_{n-1}^{(i)}+a_{n}^{(m-1)}-\gamma_{m-1}-q\left(a_{n-1}^{(m-1)}-\gamma_{m-1}\right)}{1-\frac{n q}{n-1}} .
$$

Finally the Stolz theorem implies

$$
\lim _{n \rightarrow \infty} a_{n}^{(m)}=\lim _{n \rightarrow \infty} \frac{c_{n}-c_{n-1}}{d_{n}-d_{n-1}}=\frac{q}{p} \sum_{i=0}^{m-1}\left(\begin{array}{c}
m \\
i
\end{array}\right) \gamma_{i} .
$$

Now we are ready to prove the main result.

Proof of Theorem 1. Exploiting the recurrence definition of $\left(a_{n}^{(r)}\right)$ given in Lemma 3 one can easily see, using for instance an induction with respect to $r$, that

$$
a_{n}^{(r-1)}-\gamma_{r-1}=n^{r}\left(\sum_{i=1}^{n} \frac{q^{n-i}}{i}-\sum_{j=1}^{r} \frac{\gamma_{j-1}}{n^{j}}\right)
$$


for any $n=1,2, \ldots, r=1,2, \ldots$ Thus, it follows from Lemma 3 that

$$
\sum_{i=1}^{n} \frac{q^{n-i}}{i}=\sum_{j=1}^{r} \frac{\gamma_{j-1}}{n^{j}}+o\left(1 / n^{r}\right)
$$

for any $r=1,2, \ldots$. Now the final result concerning convergence is a consequence of Lemma 2, while the form of coefficients follows from the uniqueness of the recurrence relation (4) for the Eulerian polynomials, since $\gamma_{0}=1 / p=A_{0}(q) / p$, i.e. $\gamma_{j}=$ $A_{j}(q) / p^{j+1}, j=1,2, \ldots$.

\section{ACKNOWLEDGEMENT}

Due to referee's remarks the exposition of the material covered in the paper has been considerably improved.

\section{REFERENCES}

Chao, M.Y., Strawderman, W.E. (1972) Negative moments of positive random variables. Journal of the American Statistical Association 67, 429-431.

Cochran, W.G. (1977) Sampling Techniques. Wiley, New York. MR 14:887i

Cressie, N.A.C., Davis, A.S., Folks, J.L., Policello, G.E. (1981) The moment-generating function and negative integer moments. American Statistician 35, 148-150. MR 82j:62009

David, F.N., Johnson, N.L. (1956-7) Reciprocal Bernoulli and Poisson variables. Metron 18, 77-81. MR 18:520g

Désarménien, J., Foata, D. (1992) The signed Eulerian numbers. Discrete Mathematics 99, 49-58. MR 93e:11030a

Fonta, D., Schützenberger, M.-P. (1970) Théorie Géométrique des Polynômes Eulériens. Lecture Notes in Mathematics 138, Berlin, Springer. MR 42:7523

Govindarajulu, Z. (1963) Recurrence relations for the inverse moments of the positive binomial variable. Journal of the American Statistical Association 58, 468-473. MR 26:7068

Grab, E.L., Savage, I.R. (1954) Tables of the expected value of $1 / x$ for positive Bernoulli and Poisson variables. Journal of the American Statistical Association 49, 169-177. MR 15:636g Graham, R.L., Knuth, D.E., Patashnik, O. (1994) Concrete Mathematics. A Foundation of Computer Science, Addison-Wesley, Reading, Massachusetts. MR 91f:00001

Jones. M.C. (1987) Inverse factorial moments. Statistics and Probability Letters 6, 37-42. MR 90e:62025a

KABE, D.G. (1976) Inverse moments of discrete distributions. Canadian Journal of Statistics 4, 133-141. MR 56:6961

Mendenhall, W., Lehman, JR., E.H. (1960) An approximation to the negative moments of the positive binomial useful in life testing. Technometrics 2, 227-242. MR 22:5103

Patel, M.N., GajJar, A.V. (1995) Some results on maximum likelihood estimators of parameters of exponential distribution under type I progressive censoring with changing failure rates. Communications in Statistics. Theory and Methods 24, 2421-2435. MR 96h:62179

Rempala, G., SzéKely, G.J. (1998) On estimation with elementary symmetric polynomials. Random Operations and Stochastic Equations 6, 77-88. CMP 98:09

Rockower, E.B. (1988) Integral identities for random variables. American Statistician 42, 6872. MR 89a:60041

Stancu, D.D. (1968) On the moments of negative order of the positive Bernoulli and Poisson variables. Studia Universitatis Babes Bolyai Series 13, 27-31. MR 37:2352

Stephan. F.F. (1946) The expected value and variance of the reciprocal and other negative powers of a positive Bernoullian variate. Annals of Mathematical Statistics 16, 50-61. MR 6:232h

SzabŁowski, P.J., WesoŁowski, J., Wieczorkowski, R. (1996), Estymacja w podpopulacjach. Wiadomości Statystyczne 41(7), 1-13 (in Polish).

SzabŁowski, P.J., WesoŁowski, J., Wieczorkowski, R. (1997), Estimation in subpopulations. Bulletin of the International Statistical Institute 57(2) (51st Session, Contributed Papers), 365366. 
Thionet, P. (1963) Sur le moment d'ordre $(-1)$ de la distribution tronqueé. Application a l'échantillonage de Hajek. Publ. Inst. Statist. Univ. Paris 12, 93-102. MR 31:827

Tiago de Oliveira, J. (1952) Sur le calcul des moments de la réciproque d'une varaible aléatoire positive de Bernoulli et Poisson. Anais da Faculdade de Ciencias do Porto 36, 165-168. MR 15:969c

Mathematical Institute, Warsaw University of Technology, Plac Politechniki 1, 00-661 Warsaw, Poland

E-mail address: wesolo@alpha.im.pw.edu.pl 